\title{
2-(trimethylammonium)ethyl (R)-3-methoxy-3-oxo-2-stearamidopropyl phosphate enhances thrombopoietin-induced megakaryocytic differentiation and plateletogenesis
}

\author{
Jusong Kim ${ }^{1}$, Guanghai Jin ${ }^{2}$, Jisu Lee ${ }^{2}$, Kyeong Lee ${ }^{2, *}$, Yun Soo Bae ${ }^{1, *} \mathcal{E}$ Jaesang Kim $^{1, *}$ \\ ${ }^{1}$ Department of Life Science, Ewha Womans University, Seoul 03760, ${ }^{2}$ College of Pharmacy, Dongguk University, Goyang 10326, Korea
}

\begin{abstract}
We have previously reported the effects of 2-(trimethylammonium)ethyl (R)-3-methoxy-3-oxo-2-stearamidopropyl phosphate [(R)-TEMOSPho], a synthetic phospholipid, on megakaryocytic differentiation of myeloid leukemia cells. Here, we demonstrate that $(R)$-TEMOSPho enhances megakaryopoiesis and plateletogenesis from primary hematopoietic stem cells (HSCs) induced by thrombopoietin (TPO). Specifically, we demonstrate at sub-saturation levels of TPO, the addition of $(R)$-TEMOSPho enhances differentiation and maturation of megakaryocytes (MKs) from murine HSCs derived from fetal liver. Furthermore, we show that production of platelets with $(R)$-TEMOSPho in combination with TPO is also more efficient than TPO alone and that platelets generated in vitro with these two agents are as functional as those from TPO alone. TPO can thus be partly replaced by or supplemented with $(R)$-TEMOSPho, and this in turn implies that $(R)$-TEMOSPho can be useful in efficient platelet production in vitro and potentially be a valuable option in designing cell-based therapy. [BMB Reports 2019; 52(7): 434-438]
\end{abstract}

\section{INTRODUCTION}

Platelets are anucleate cellular fragments found in large numbers in circulating blood (1). They originate from MKs which have descended from HSCs and undergo endomitosis achieving polyploidy of upto $256 \mathrm{~N}$ prior to apoptotic disintegration and release of platelets $(2,3)$. Thrombosis and hemostasis are both

${ }^{*}$ Corresponding authors. Kyeong Lee, Tel: +82-31-961-5214; Fax: +82-31-961-5206; E-mail: kaylee@dongguk.edu; Yun Soo Bae, Tel: +82-2-3277-2729; Fax: +82-2-3277-3760; E-mail: baeys@ewha. ac.kr; Jaesang Kim, Tel: +82-2-3277-3414; Fax: +82-2-3277-3760; E-mail: jkim1964@ewha.ac.kr

https://doi.org/10.5483/BMBRep.2019.52.7.200

Received 29 August 2018, Revised 28 September 2018, Accepted 12 October 2018

Keywords: (R)-TEMOSPho, Megakaryocyte, Platelet, Thrombopoietin mediated by platelets whose well balanced activity is thus crucial for vascular integrity and ultimately for survival. Platelets, currently obtained from donated blood, are used in various medical procedures including cancer treatments and certain types of surgery. Not surprisingly, not only is there a widespread shortage problem, but side effects such as febrile and anaphylactoid reactions and blood-borne infections remain as unresolved risk factors (4).

Numerous efforts to generate platelets in vitro have been made, and most of recent attempts are based essentially on a sequential differentiation of embryonic stem cells (ESCs) or induced pluripotent stem cells (iPSCs) first to HSCs and then to polyploidy MKs which can subsequently undergo apoptosis and release platelets (5-8). The key reagent for both the generation of HSCs and the induction of megakaryocytic differentiation is TPO. The need for this humoral growth factor and its receptor, CD110 encoded by c-mpl gene, in generation of platelets via maturation of MKs is well demonstrated both in vitro and in vivo $(9,10)$. Indeed, virtually all procedures reporting in vitro platelet generation to date use TPO as the essential element (5-8).

Generation and purification of large quantities of TPO suitable for application in cell culture-based production is likely to be highly costly. Here, we describe an alternate strategy utilizing $(R)$-TEMOSPho, a synthetic chemical previously shown to induce megakaryocytic differentiation of myeloid leukemia cells, as a supplement to TPO $(11,12)$. Specifically, we show that $(R)$-TEMOSPho, which cannot by itself induce efficient MK differentiation of murine HSCs, can enhance TPO activity in megakaryocytic differentiation and plateletogenesis. This implies that $(R)$-TEMOSPho can potentially be a useful reagent in efficient in vitro production of platelets and possibly in therapeutic settings.

\section{RESULTS AND DISCUSSION}

We have previously shown that (R)-TEMOSPho can efficiently induce megakaryocytic differentiation of K562 and HEL cells which are myeloid leukemia cells (12). In order to examine its function in primary cells, we applied (R)-TEMOSPho to murine

ISSN: 1976-670X (electronic edition)

Copyright (C) 2019 by the The Korean Society for Biochemistry and Molecular Biology

c) This is an open-access article distributed under the terms of the Creative Commons Attribution Non-Commercial License (http://creativecommons.org/licenses/by-nc/4.0) which permits unrestricted non-commercial use, distribution, and reproduction in any medium, provided the original work is properly cited. 
embryonic HSCs prepared from E12.5 fetal liver. Although a slight increase in the proportion of CD41(+) cells was noted, the induction of megakaryocytic differentiation was overall negligible (Fig. 1A). Furthermore, the inductive effect on CD41 expression was not enhanced at higher levels of $(R)$-TEMOSPho. In fact, beyond $50 \mu \mathrm{g} / \mathrm{ml}$ of the reagent, we noted cell death, a
A

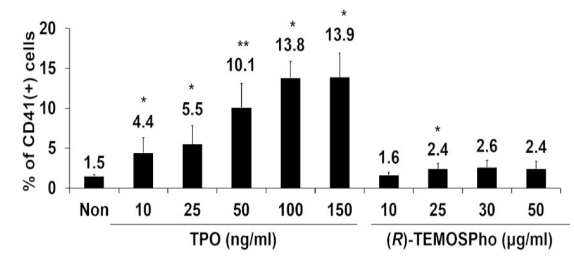

B

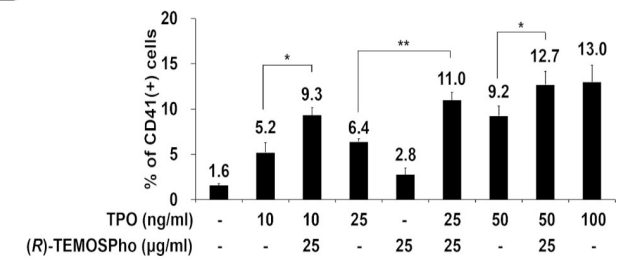

C

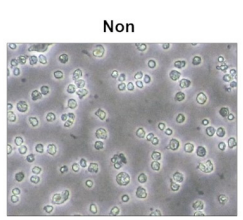

TPO $25 \mathrm{ng} / \mathrm{ml}$

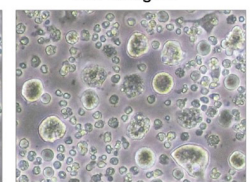

(R)-TEMOSPho $25 \mu \mathrm{g} / \mathrm{ml}$

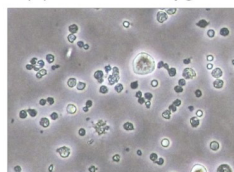

TPO $25 \mathrm{ng} / \mathrm{ml}$
$+(R)$-TEMOSPho $25 \mu \mathrm{g} / \mathrm{ml} \quad$ TPO $100 \mathrm{ng} / \mathrm{ml}$
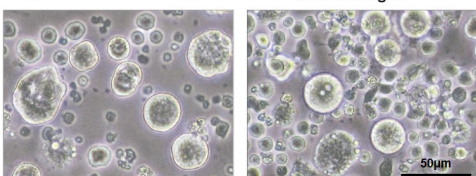

Fig. 1. Promotion of TPO-induced megakaryocytic differentiation by (R)-TEMOSPho. (A) Dose-dependent induction of CD41 as a megakaryocytic marker by TPO treatment for 5 days is shown. In contrast, $(R)$-TEMOSPho by itself has virtually no effect. (B) Combinatorial application of TPO and (R)-TEMOSPho efficiently induces CD41 expression. Note that at all concentrations (ng/ml) of TPO tested, addition of $25 \mu \mathrm{g} / \mathrm{ml}$ of (R)-TEMOSPho induces CD41 to higher levels than TPO alone. (C) Phase contrast micrographs of cells with indicated treatments. Note that the combination of TPO and $(R)$-TEMOSPho has more pronounced effect in inducing cellular enlargement, a hallmark feature of megakaryocytic differentiation than TPO alone while (R)-TEMOSPho by itself has little effect. Statistical significance, tested by Student's t-test is indicated ( $* P<0.05, * * P<0.005)$.

A
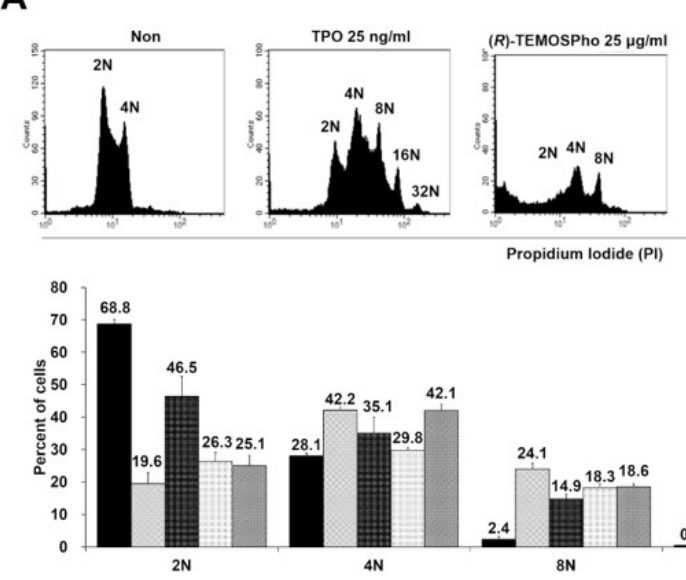

Propidium lodide (PI)

TPO $25 \mathrm{ng} / \mathrm{ml}$

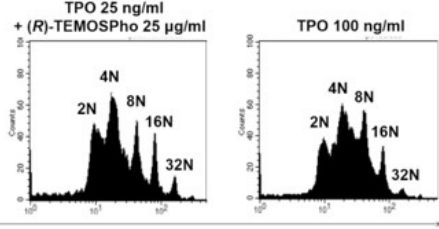

- Non $25 \mathrm{ng} / \mathrm{ml}$

-TPO $25 \mathrm{ng} / \mathrm{ml}$

a (R)-TEMOSPho $25 \mu \mathrm{g} / \mathrm{ml}$
पPO $25 \mathrm{ng} / \mathrm{ml}+$ (R)-TEMO $25 \mu \mathrm{g} / \mathrm{ml}$
ॠTPO $100 \mathrm{ng} / \mathrm{ml}$

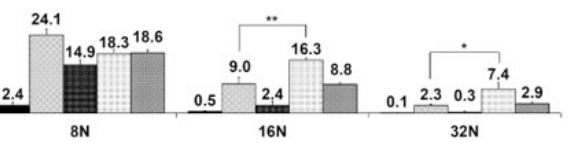

B

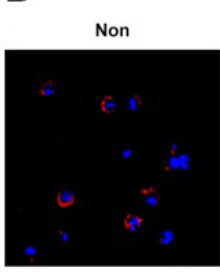

TPO $25 \mathrm{ng} / \mathrm{ml}$

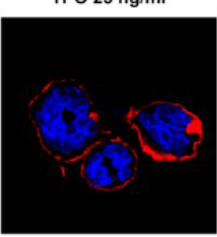

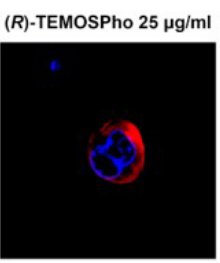

TPO $25 \mathrm{ng} / \mathrm{ml}$
R)-TEMOSPho $25 \mu \mathrm{g} / \mathrm{m}$

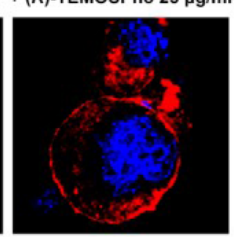

TPO $100 \mathrm{ng} / \mathrm{ml}$

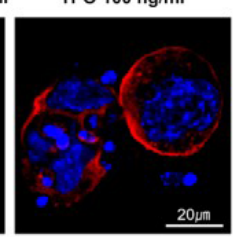

Fig. 2. Promotion of TPO-induced polyploidy by (R)-TEMOSPho. (A) Flow cytometric analyses for DNA contents following indicated treatments. Note that the combination of TPO and (R)TEMOSPho has more pronounced effect in inducing polyploidy, another hallmark feature of megakaryocytic differentiation than TPO alone. In the graph at the bottom, relative proportions of populations with distinct polyploidy are shown. Note the higher proportions of $16 \mathrm{~N}$ and $32 \mathrm{~N}$ populations among cells treated with both TPO and (R)-TEMOSPho than among cells treated with TPO alone. The results represent mean \pm SD from three independent assays. (B) Cells were DAPI-stained (blue) for nuclei and Factin-stained (red) with phalloidin to delineate boundaries of single cells. Statistical significance, tested by Student's t-test is indicated $(* \mathrm{P}<$ $0.05, * * \mathrm{P}<0.005)$. 
likely side effect of high dosage. This was in contrast to the effect of TPO which induced CD41 expression in a dose dependent manner maximally around $100 \mathrm{ng} / \mathrm{ml}$ (Fig. 1A). We were curious to see if $(R)$-TEMOSPho could nevertheless contribute to megakaryocytic differentiation by enhancing the effect of TPO. (R)-TEMOSPho and sub-maximal doses of TPO were applied in combination, and CD41 expression was examined. Interestingly, $25 \mu \mathrm{g} / \mathrm{ml}$ of $(R)$-TEMOSPho in combination with 25 or $50 \mathrm{ng} / \mathrm{ml}$ of TPO could induce the proportions of $\mathrm{CD} 41(+)$ cells to the near maximal level achieved by $100 \mathrm{ng} / \mathrm{ml}$ of TPO (Fig. 1B). The bona fide induction of megakaryocytic differentiation was also confirmed by visible increase in cellular size (Fig. 1C).

Another hallmark phenotype associated with megakaryopoiesis is endomitosis and resulting polyploidy. Indeed, TPO treatment readily induced increase in chromosomal content (Fig. 2A). As in the case of cellular enlargement, supplementing submaximal level of TPO (25 ng/ml) with (R)-TEMOSPho promoted polyploidization. Notably, cells with higher chromosomal contents of $16 \mathrm{~N}$ and $32 \mathrm{~N}$ were relatively enriched among cells treated with both TPO and (R)-TEMOSPho even when compared to cells treated with $100 \mathrm{ng} / \mathrm{ml}$ of TPO. The increase in chromosomal content was also confirmed via fluorescence microscopy with DAPI-staining for chromosomes and F-actin staining to delineate cellular boundaries (Fig. 2B).

We next investigated whether culture-derived MKs can produce platelets and if (R)-TEMOSPho can aid TPO in the process. Proplatelet-like projections were readily seen in TPOtreated cultures, and supplementing with (R)-TEMOSPho appeared to enhance the level induced by the sub-maximal concentration of TPO (Fig. 3A). In order to quantify the plateletogenesis, we followed a previously established protocol for isolating platelets from mouse sera based on forward and side scatter properties and assessing the platelet level based on

A
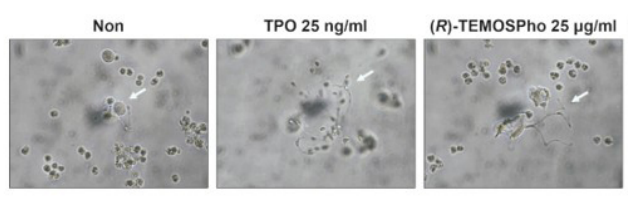

TPO $25 \mathrm{ng} / \mathrm{m}$
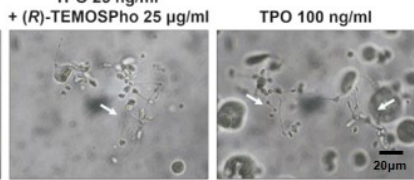

B
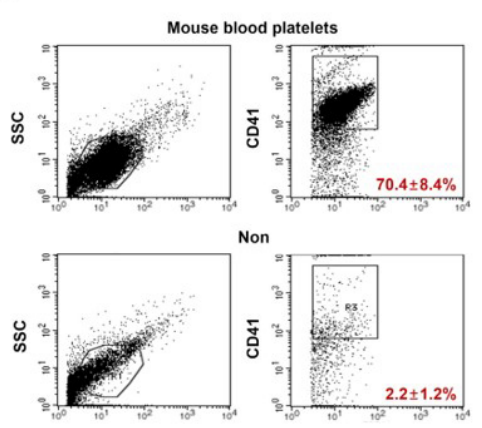

TPO $25 \mathrm{ng} / \mathrm{m}$
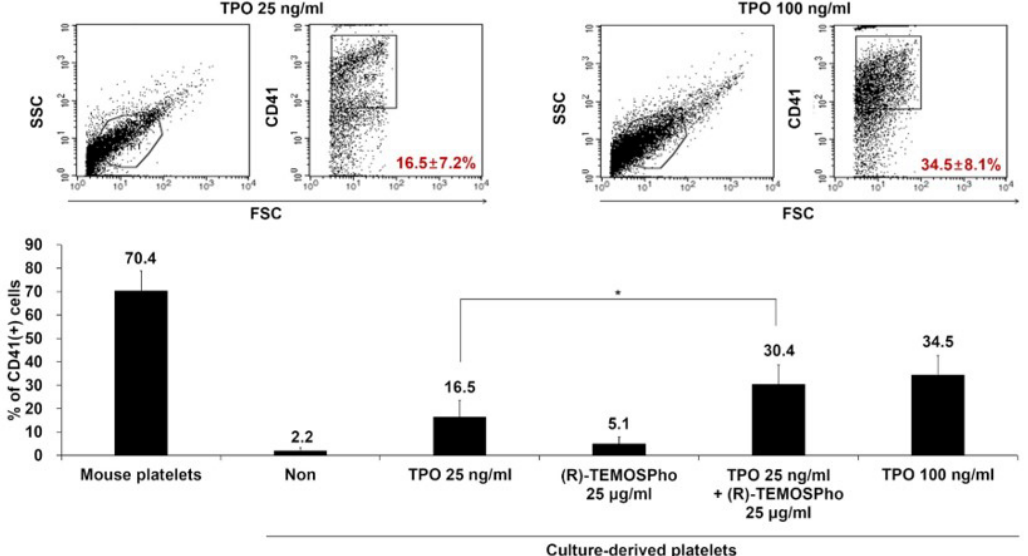

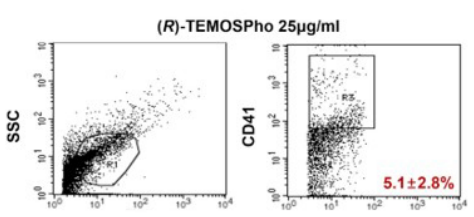

TPO $25 \mathrm{ng} / \mathrm{ml}+(R)$-TEMOSPho $25 \mu \mathrm{g} / \mathrm{ml}$

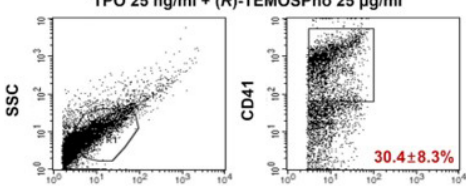

TPO $100 \mathrm{ng} / \mathrm{ml}$

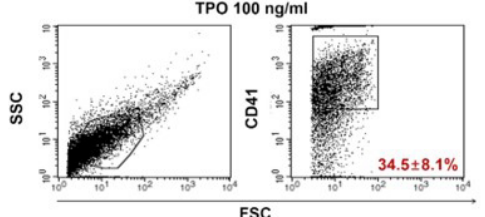

Culture-derived platele
Fig. 3. Promotion of TPO-induced plateletogenesis by (R)-TEMOSPho. (A) Phase contrast micrographs of proplatelet projections (white arrows) are shown. Cells were cultured for 10 days in vitro. Note that virtually no proplatelet projections are seen with $(R)$-TEMOSPho alone. In contrast, TPO treatment with or without $(R)$-TEMOSPho generated proplatelet projections which were readily confirmed by visual inspection. (B) Platelets isolation from mouse blood or culture media are shown. Platelets were isolated by forward and side scatter and quantitated based on forward scatted and CD41 expression. Results are shown in the graph below. Note higher proportions of CD41 positive platelets present in culture media of cells treated with both TPO and $(R)$ TEMOSPho than in the culture media cells treated with $25 \mathrm{ng} / \mathrm{ml}$ of TPO alone. The results represent mean \pm $\mathrm{SD}$ from three independent assays. Statistical significance, tested by Student's t-test is indicated ( $* P<0.05)$. 
CD41 expression $(5,12,13)$. Using mouse blood-derived platelet preparation as the positive control, we isolated platelet populations with identical flow cytometric characteristics. The proportions of CD41(+) cellular particles clearly indicated that supplementing with $(R)$-TEMOSPho significantly stimulated the plateletogenesis induced by TPO (Fig. 3B).

Next, we examined whether culture-derived platelets are functional. Mouse platelets described as above were used as the positive control. First, we investigated the expression of P-selectin, a cell adhesion molecule on the surface of activated platelets. Upon stimulation with the agonist peptide AYPGKF, platelets prepared from various conditions showed positive response to P-selectin by flow cytometric analyses consistent with activation of PAR4 receptor activation and translocation of P-selectin to the cell surface (Fig. 4A). We also examined fibrinogen-binding activity. Again, upon activation with the peptide AYPGKF, a strong binding to fluoroprobe-coupled fibrinogen was seen for culture-derived platelets as well as for mouse blood-derived platelets (Fig. 4B). Lastly, we visually examined the platelet aggregation upon activation. Indeed, phase-contrast microscopic examination showed clear aggregation post activation consistent with functionality of the platelets (Fig. 4C).

In sum, we have demonstrated that $(R)$-TEMOSPho promotes TPO-induced megakaryocytic differentiation and platelet production from primary hematopoietic stem cells. This makes it possible to use sub-saturation levels of TPO in generating platelets in vitro and could potentially make the process more cost-efficient and thus viable as an alternative to platelet transfusion. Furthermore, our results also suggests that $(R)$ TEMOSPho is potentially applicable in TPO-based or c-mpl agonist-based therapeutic treatments as a supplement.

\section{MATERIALS AND METHODS}

\section{Isolation and culture of HSCs}

The plan for this study was reviewed and approved by the Institutional Animal Care and Use Committee (IACUC) of Ewha Womans University (No. 16-050). Timed pregnant mice were purchased from a commercial vendor (Orientbio Inc.). Mouse fetal livers were isolated from embryonic day (E) 12.5 and turned into single cell suspension by pipetting and passing through cell strainer. After removing red blood cells, HSCs were purified using The EasySep Mouse Hematopoietic Progenitor

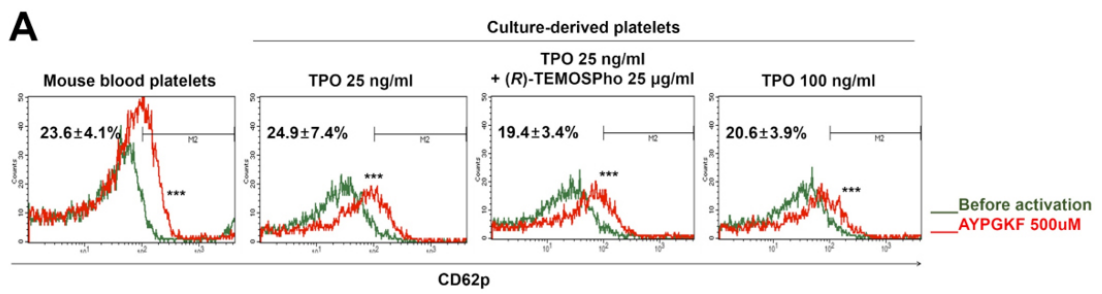

B

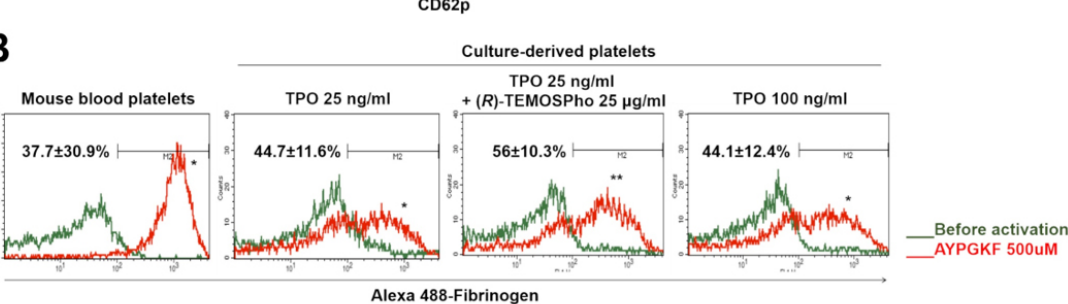

\section{C}
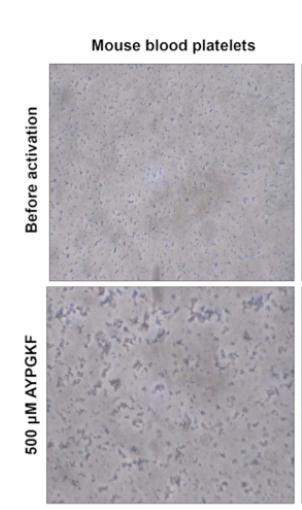

Culture-derived platelets TPO $25 \mathrm{ng} / \mathrm{ml}$
$+(R)$-TEMOSPho $25 \mu \mathrm{gg} / \mathrm{m}$

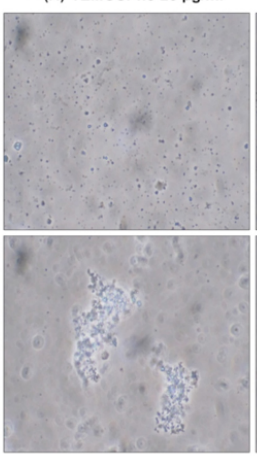

TPO $100 \mathrm{ng} / \mathrm{ml}$

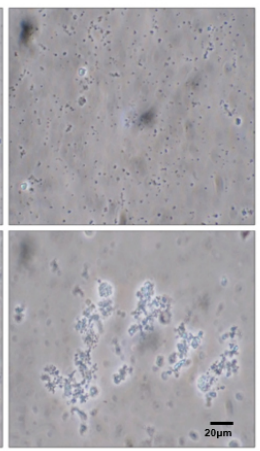

Fig. 4. Functional characterization of culture-derived platelets. (A) Platelets from mouse blood plasma and culture media were activated with the peptide AYPGKF and examined for P-selectin (CD62p) expression by flow cytometry. Green and red histograms represent naïve and activated platelets respectively. Note that all culture-derived platelets show similar level of P-selectin expression upon activation. The results represent mean $\pm S D$ from three independent assays. (B) Platelets from mouse blood plasma and culture media were activated with AYPGKF and examined for fibrinogen binding by flow cytometry. Green and red histograms represent naïve and activated platelets respectively. Note that all culture-derived platelets show similar extents of fibrinogen binding upon activation. The results represent mean \pm SD from three independent assays. (C) Aggregation upon activation was examined by phase contrast microscopy. Note the aggregation in all platelet preparations. Statistical significance, tested by Student's t-test is indicated $\left(* P<0.05, * * P<0.005,{ }^{* * * P}<\right.$ 0.0005). 
Cell Enrichment Kit (STEMCELL Technologies Inc.) following the manufacturer's protocols. Details are available upon request. Purified cells were seeded in $35 \mathrm{~mm}$ dishes $\left(\sim 2 \times 10^{5}\right)$ and cultured for 5 days in StemSpan ${ }^{\mathrm{TM}}$ Serum-Free Expansion Medium (STEMCELL Technologies Inc.). Murine TPO (Peprotech) and $(R)$-TEMOSPho were added at the indicate concentrations. (R)-TEMOSPho was synthesized following previously published steps with minor modifications (14). Details are available upon request.

\section{Flow cytometry}

Cells were incubated with FITC conjugated anti-CD41 antibody (BD biosciences) and analyzed by flow cytometry using a FACS Calibur (BD biosciences) and the BD Cell-Quest ${ }^{\text {TM }}$ Pro version 6.0 software (BD bioscience). For DNA content analysis, cells were incubate for $30 \mathrm{~min}$ at room temperature (RT) in staining solution $(50 \mu \mathrm{g} / \mathrm{ml}$ Propidium lodide, $100 \mu \mathrm{g} / \mathrm{ml}$ RNase A, $0.1 \%$ Triton X-100) and analyzed by FACS Calibur.

\section{Phalloidin staining}

Staining for F-actin with phalloidin to delineate cellular boundaries and counterstaining with 4'-6-Diamidino-2-phenylindole (DAPI) were carried out as previously described (11).

\section{Platelet functional assays}

Platelet-rich plasma was prepared from mouse blood and resuspended in Tyrode buffer following a published protocol with minor modifications $(5,13)$. Details are available upon request. For preparation of culture-derived mouse platelets, culture medium was collected and centrifuged at $150 \mathrm{~g}$ for 20 min to isolate the supernatant to which $1 \mu \mathrm{M}$ PGE1 and 0.1 $\mathrm{U} / \mathrm{ml}$ apyrase in 9X volume of ACD solution (Sigma-Aldrich) were added. After centrifugation at $900 \mathrm{~g}$ for $10 \mathrm{~min}$, the pellet was resuspended and washed with the washing solution containing $1 \mu \mathrm{M}$ PGE1 and $0.1 \mathrm{U} / \mathrm{ml}$ apyrase. The pellet containing platelets were finally isolated by centrifugation and resuspended in Tyrode buffer. To examine P-selectin expression, platelets were stimulated with $500 \mu \mathrm{M}$ AYPGKF (Peptron) and incubated for $15 \mathrm{~min}$ at $37^{\circ} \mathrm{C}$ prior to incubation with FITC conjugated anti-CD62p antibody (BD Bioscience) for $15 \mathrm{~min}$ at RT and analysis by FACS. For the fibrinogen binding assay, platelets were first activated with $500 \mu \mathrm{M}$ AYPGKF and subsequently incubated with the 3ul Alexa 488-labelled fibrinogen (Invitrogen) for $30 \mathrm{~min}$ in the dark. The mixture was then diluted with $1 \mathrm{X}$ PBS and analyzed by FACS. For the aggregation assay, platelets were activated with $100 \mu \mathrm{g} / \mathrm{ml}$ fibrinogen (Merck Millipore), $1 \mathrm{mM} \mathrm{CaCl}, 1 \mathrm{mM} \mathrm{MgCl}_{2}$ and $500 \mu \mathrm{M}$ AYPGKF and incubated for at RT for $10 \mathrm{~min}$. The activated platelets were applied to $2.5 \mathrm{mg} / \mathrm{ml}$ fibrinogen coated slide and examined for platelet aggregation under a phase-contrast microscope.

\section{ACKNOWLEDGEMENTS}

This work was supported by Stem Cell Grant (NRF- 2017M3A9 B3061850), Aging Project Grant (NRF-2017M3A9D8062955) and Innovative Medicine Research Center for Tumor Remission (NRF-2018R1A5A2023127) from the National Research Foundation of Korea funded by the Ministry of Science and ICT, Republic of Korea. This study was also supported by the Ewha Womans University scholarship of 2018.

\section{CONFLICTS OF INTEREST}

The authors have no conflicting interests.

\section{REFERENCES}

1. Michelson AD (2003) How platelets work: platelet function and dysfunction. J Thromb and Thrombolysis 16, 7-12

2. Deutsch VR and Tomer A (2006) Megakaryocyte development and platelet production. Br J Haematol 134, 453-466

3. Kaushansky K (2008) Historical review: megakaryopoiesis and thrombopoiesis. Blood 111, 981-986

4. Kiefel V (2008) Reactions Induced by Platelet Transfusions. Transfus Med Hemother 35, 354-358

5. Fujimoto TT, Kohata S, Suzuki H, Miyazaki $\mathrm{H}$ and Fujimura K (2003) Production of functional platelets by differentiated embryonic stem (ES) cells in vitro. Blood 102, 4044-4051

6. Lu SJ, Li F, Yin H et al (2011) Platelets generated from human embryonic stem cells are functional in vitro and in the microcirculation of living mice. Cell Res 21, 530-545

7. Moreau T, Evans AL, Vasquez L et al (2016) Large-scale production of megakaryocytes from human pluripotent stem cells by chemically defined forward programming. Nat Commun 7, 11208

8. Takayama N, Nishikii H, Usui J et al (2008) Generation of functional platelets from human embryonic stem cells in vitro via ES-sacs, VEGF-promoted structures that concentrate hematopoietic progenitors. Blood 111, 5298-5306

9. Kaushansky K (2016) Thrombopoietin and its receptor in normal and neoplastic hematopoiesis. Thromb J 14, 40

10. Kaushansky K and Drachman JG (2002) The molecular and cellular biology of thrombopoietin: the primary regulator of platelet production. Oncogene 21, 3359-3367

11. Han SH, Kim J, Her Y et al (2015) Phytosphingosine promotes megakaryocytic differentiation of myeloid leukemia cells. BMB Rep 48, 691-695

12. Limb JK, Song D, Jeon M et al (2015) 2-(trimethylammonium)ethyl (R)-3-methoxy-3-oxo-2-stearamidopropyl phosphate promotes megakaryocytic differentiation of myeloid leukaemia cells and primary human CD34(+) haematopoietic stem cells. J Tissue Eng Regen Med 9, 435-446

13. Opalinska JB, Bersenev A, Zhang Z et al (2010) MicroRNA expression in maturing murine megakaryocytes. Blood 116, e128-138

14. Kim YA, Chung HM, Park JS et al (2003) Synthesis of novel lysophosphatidylcholine analogues using serine as chiral template. J Org Chem 68, 10162-10165 